\title{
Effects of forced swimming stress on expression and phosphorylation of PI3K/Akt signal pathway in pancreas of type 2 diabetic rats
}

\author{
Lan $\mathrm{Li}^{1 \#}$, Dan $\mathrm{He}^{2 \#}$, Kewei Jiang ${ }^{1}$, Ying Zhao ${ }^{1}$ \\ ${ }^{1}$ Department of Geriatrics, Shenzhen People's Hospital, The First Affiliated Hospital of Southern University of Science and Technology, The \\ Second Clinical Medical College of Jinan University, Shenzhen, China; ${ }^{2}$ Department of Endocrinology and Metabolism, Xiangtan Central Hospital, \\ Xiangtan, China \\ Contributions: (I) Conception and design: L Li, D He; (II) Administrative support: All authors; (III) Provision of study materials or patients: All \\ authors; (IV) Collection and assembly of data: All authors; (V) Data analysis and interpretation: L Li, D He; (VI) Manuscript writing: All authors; (VII) \\ Final approval of manuscript: All authors. \\ \#The authors contributed equally to this work. \\ Correspondence to: Lan Li. Department of Geriatrics, Shenzhen People's Hospital, The First Affiliated Hospital of Southern University of Science and \\ Technology, The Second Clinical Medical College of Jinan University, No.1017 Dongmen North Road, Luohu District, Shenzhen, China. Email: \\ doctorlilan@aliyun.com.
}

Background: To study the effects of forced swimming stress on blood glucose and insulin levels and the expression and phosphorylation of pancreatic PI3K/Akt signal pathway in type 2 diabetic rats.

Methods: Thirty adult SD rats (8-week-old, male) were randomly divided into three groups: control group, diabetic model group, and diabetic model stress group. The diabetic model group was established by feeding with the high-fat and high-glucose diet for four weeks, and then the rats were injected with low-dose streptozotocin (STZ, $25 \mathrm{mg} / \mathrm{kg}$, once a day for 2 days). The rats in the diabetic model group were subjected to forced swimming stress for seven days, which was a diabetic model stress group. Twenty-four hours after the last forced swimming stress, the rats fasted, and blood samples were collected to detect blood glucose, insulin, triglycerides (TGs), free fatty acids (FFAs), high- and low-density lipoprotein cholesterol (HDLC and LDLC) levels. The western blot was applied to detect the expression of PI3K, Akt, p-Akt, and mTOR in the pancreas of rats in each group.

Results: Blood glucose and insulin levels in the diabetic model stress group were significantly lower than those in the diabetic model group, and the protein levels of PI3K, p-AKT, and mTOR in the pancreas of the diabetic model stress group were significantly higher than those of the diabetic model group.

Conclusions: One-week swimming stress can decrease the blood glucose level and improve insulin indexes in type 2 diabetic rats and increase the expression and phosphorylation of pancreatic PI3K/Akt signal pathway proteins.

Keywords: Type 2 diabetes mellitus (T2DM); forced swimming; stress; phosphatidylinositol 3 kinase; protein kinase B

Submitted Jun 23, 2020. Accepted for publication Aug 05, 2020.

doi: $10.21037 / \mathrm{atm}-20-5304$

View this article at: http://dx.doi.org/10.21037/atm-20-5304 


\section{Introduction}

In the past decades, the number of people who have diabetes has been increasing, spreading all over the world. It is supposed the number of people troubled with diabetes will increase by more than $50 \%$ from 2017 to 2045, resulting in about 693 million people with diabetes, and the annual medical cost will be about $\$ 850$ billion (1). The prevalence of diabetes is due to the increased incidence of type 2 diabetes mellitus (T2DM), characterized by inadequate insulin secretion and insulin resistance (2). Lifestyle changes induced the increase of high-energy, refined food intake, sedentary behavior, and other risk factors, including environmental pollution, socio-economic and psychological conditions, like smoking and sleep problems, play an essential part in the occurrence and development of type 2 diabetes (3-5). It has proved that stress is an essential factor in the pathogenesis of type 2 diabetes. Repeated or continuous stress exposure can lead to dysfunction of glucose metabolism, functional nerve-endocrine disorder, and chronic inflammation, which can predict the occurrence of T2DM (6). Patients with diabetes also have neuroendocrine disorders under stress, including increased glucocorticoid and auxin levels, decreased luteinizing hormone, and follicle-stimulating hormone levels (7).

Moreover, due to the inconvenience of stress operation and other reasons, the effect of stress on the physiological indexes of patients with T2DM and its mechanism is still unclear. Forced swimming is a commonly used stress model for the modeling and detection of depression (8). The diabetic model of a high-fat diet with streptozotocin (STZ) is remarkably like human type 2 diabetes (9). Therefore, in this work, the rats were induced to develop type 2 diabetes by fed with a high-fat diet and injected with low-dose STZ (10). Also, the effects of forced swimming stress on the protein expression and phosphorylation of the PI3K/ Akt signal pathway in the pancreas of diabetic rats were observed to explore the role and molecular mechanism of stress in T2DM.

We present the following article in accordance with the ARRIVE reporting checklist (available at http://dx.doi. org/10.21037/atm-20-5304).

\section{Methods}

\section{Primary reagents and equipment}

PI3K antibody (abcam, ab151549), AKT antibody (abcam, ab8805), p-Akt antibody (Cell Signaling Technology, 4060T),
mTOR antibody (abcam, ab32028), $\beta$-Actin antibody (abcam, ab179467), Goat anti-Rabbit IgG-HRP (Bioworld); Radio Immunoprecipitation Assay (RIPA) Buffer (CST, \#9806S), Protease Inhibitor Cocktail Set I (CALBIOCHEM, \#539131), BCA protein concentration quantitative kit (Pierce, \#23225); blood glucose, triglyceride (TG), free fatty acid (FFA), highand low-density lipoprotein cholesterol (HDLC and LDLC) kit (Nanjing Jiancheng Biological Co., Ltd., China), insulin kit (Shanghai Shenggong Biotechnology Service Co., Ltd., China); Desktop freezing centrifuge, UV spectrophotometer, enzyme labeling instrument, electrophoresis instrument (Tianneng EPS-300), vertical electrophoresis tank (Tianneng VE-180), transfer electrophoresis tank (Tianneng VE-186), $\mathrm{X}$-ray cassette (AX-II).

\section{Animal grouping and modeling}

Thirty healthy male SD rats, aged 8 weeks, weighed $250 \pm 15 \mathrm{~g}$ (bought from Guangdong Medical Animal Center). The experimental rats were fed at $24 \pm 1^{\circ} \mathrm{C}$ for 12 hours with a light-dark cycle. The rats were fed one week for adaptation; the experimental rats were randomly divided into three groups ( $\mathrm{n}=10$ in each group): normal housed control group, diabetic model group and the diabetic model group with forced swimming stress. The no-treated control group was fed with normal diet (standard GB 14924.3-2010), and the diabetic model group and the forced swimming stress combined diabetic model group were fed with high-fat diet (10\% cooked lard, $20 \%$ sucrose, $2.5 \%$ cholesterol, $1.0 \%$ cholate and $66.5 \%$ normal diet). After four weeks of feeding, all rats fasted for 12 hours. Rats in diabetic model group and diabetic stress group were intraperitoneally injected with lowdose STZ (25 mg/kg, once a day for two consecutive days) to establish a T2DM rat model, while rats in normal control groups were injected with the same amount of normal saline. After the establishment of the model, the rats in the diabetic model stress group were given forced swimming stress for seven days. The rats swam in a glass tank with a water temperature of $24^{\circ} \mathrm{C}$ and water depth of $50 \mathrm{~cm}$ for 30 minutes daily, and then they were put back into the original cage. All animal experiments were performed in accordance with the guidelines for animal care and was approved by the Medical Ethics Committee of Shenzhen People's Hospital (No. LL-KY-2020293).

\section{Sample collection and detection}

The rats in the diabetic model stress group rested for 
24 hours after the last stimulation, and the rats in each group fasted for 12 hours. We take the blood samples from the tail vein, and the levels of fasting blood glucose (FBG) and insulin (FINS) are measured according to the kit instructions. Rat insulin sensitivity index (ISI) can be calculated by ISI $=\ln [1 / \mathrm{FBG} \times$ FINS $]$. Each rat was intragastrically administrated with $20 \%$ glucose solution according to $2 \mathrm{~g} / \mathrm{kg}$ body weight, after $120 \mathrm{mins}$, the blood samples, taken from the tail vein, were used to determine the blood glucose and insulin level. The insulin resistance index (HOME-IR) was calculated by HOME-IR = fasting blood glucose level $(\mathrm{FPG}, \mathrm{mmol} / \mathrm{L}) \times$ fasting insulin level (FINS, $\mu \mathrm{U} / \mathrm{mL}$ )/22.5. At the end of the experiment, $2 \%$ pentobarbital sodium was injected intraperitoneally at the dose of $50 \mathrm{mg} / \mathrm{kg}$ to give full anesthesia. Then blood samples harvested from the abdominal aorta were taken for TG, FFA, HDLC and LDLC test. The pancreas was isolated at once after blood collection and stored at $-80{ }^{\circ} \mathrm{C}$ for the western blot.

\section{Western blot}

An appropriate amount of the pancreatic tissue was placed on ice, an electronic balance weighed the tissue weight, and the precooled RIPA strong lytic solution $(50 \mathrm{mM}$ Tris, $\mathrm{pH}$ 7.4; $150 \mathrm{mM} \mathrm{NaCl}, 1 \%$ sodium deoxycholate; $0.1 \%$ SDS, $1 \%$ Triton X-100) containing 1\% Phenylmethylsulfonyl fluoride (PMSF) was added according to 1:5 (weight/ volume). After this, the tissue with the lytic solution was put into a glass homogenizer to break and obtain the homogenate. The homogenate was suspended in a refrigerator at $4{ }^{\circ} \mathrm{C}$ for half an hour to lyse fully, and then the homogenate was centrifuged at $4{ }^{\circ} \mathrm{C}$ and $16,000 \mathrm{~g}$ for 20 minutes, the supernatant, which contained the extracted tissue total protein, was taken. BCA protein concentration quantitative kit was used to detect the protein concentration of each sample. Each sample took the same amount of protein $(40 \mu \mathrm{g})$ for $10 \%$ SDS-PAGE electrophoresis, After electrophoresis separation, the separated protein was transferred into nitrocellulose membrane $(320 \mathrm{~mA}$ for $3 \mathrm{~h}$ ), and then the membrane was sealed with $5 \%$ skimmed milk powder (TBST dispensing) at room temperature for 1 hour, and with the TBST buffer three times. The antibody against the target protein or internal reference protein (TBST prepared 5\% BSA, added the antibody ratio 1:1,000) was added, and then the membrane was shaken overnight in the refrigerator at $4{ }^{\circ} \mathrm{C}$. The antibody was recovered, and subsequently, the membrane was washed three times with TBST. The secondary antibody was prepared with $5 \%$ skimmed milk powder (antibody ratio 1:5,000), and $5 \mathrm{~mL}$ of them was added to each membrane. The membrane was incubated with the milk described above at room temperature for 1 hour and then washed three times with TBST. The washed membrane was taken to the darkroom and mixed with electrochemiluminescence (ECL) luminous solution in the same proportion. The fluorescence signal was collected on the film by film exposure. The relevant protein content was represented by the gray value of the color strip on the film. AlphaEaseFC software analyzed the gray value, and the ratio of the gray value of the target protein strip to the gray value of its corresponding internal reference was used to reflect the relative content of the protein.

\section{Statistical analysis}

The mean \pm standard deviation expressed the experimental results $(\bar{x} \pm S D)$. SPSS 21.0 software analyzed the data. The differences between groups were evaluated by one-way analysis of variance (One-way ANOVA). The LSD method performs a pairwise comparison.

\section{Results}

\section{Blood glucose and insulin levels in fasted rats}

A diabetic rat model was constructed and given a week of forced swimming stimulation to study the effect of stress on rats suffering from type 2 diabetes. The rats were grouped and treated, as shown in Figure 1A. The results of the fasting blood glucose experiment (Figure 1B) showed the blood glucose levels in the model group, and the model stress group were significantly increased compared to the control group, indicating that the model of type 2 diabetes was successful. Also, the blood glucose level in the model stress group was significantly lower than that in the model group, which suggested that the level of blood glucose regulation in the model stress group was improved. Through the detection of insulin level, it was found the blood insulin level of the model group was significantly increased compared to the control group, while the blood insulin level of the model stress group showed a rising trend, which has no significant differences with the control group and the model group. Furthermore, the ISI (Table 1) and the insulin resistance index (HOME-IR, Table 2) of the three groups were calculated. These results suggest that compared with the control group, the ISI decreased, and 


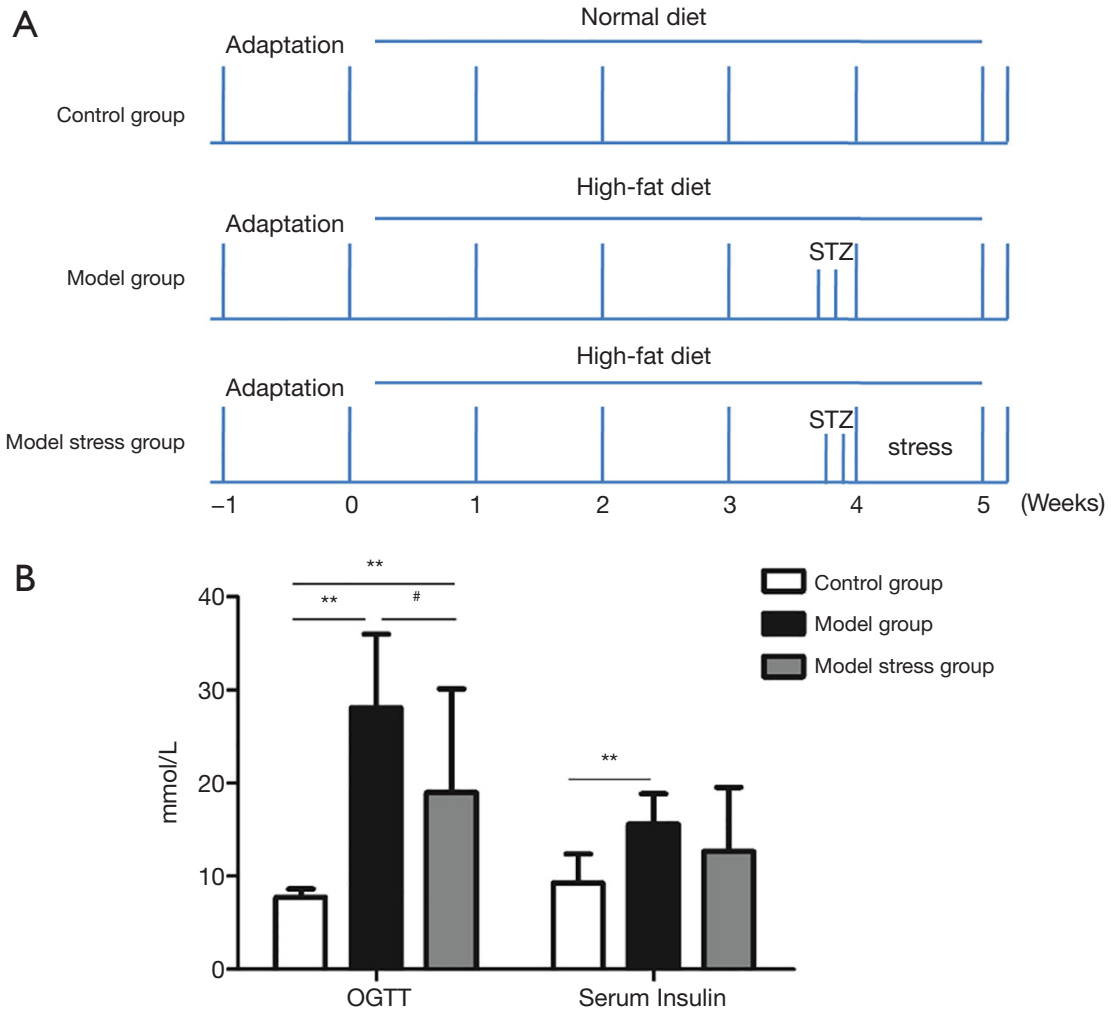

Figure 1 Construction of diabetic rat model. (A) Group and treatment of rats; (B) the concentration of blood glucose after oral glucose tolerance test (OGTT) and serum insulin. **, $\mathrm{P}<0.01$ compared to the control group; ${ }^{\#}, \mathrm{P}<0.05$ compared to the model group. STZ, streptozotocin.

Table 1 The insulin sensitivity index (ISI) of each group

\begin{tabular}{lccc}
\hline Group & Control group & Model group & Model stress group \\
\hline ISI & -4.2759 & -6.0748 & -5.4727 \\
\hline
\end{tabular}

Table 2 The insulin resistance index (HOME-IR) of each group

\begin{tabular}{lccc}
\hline Group & Control group & Model group & Model stress group \\
\hline HOME-IR & 1.4851 & 9.1717 & 4.9762 \\
\hline
\end{tabular}

the insulin resistance index increased in the model group, while the rats in the model stress group had improved in ISI and HOME-IR. One week of forced swimming stimulation can improve the blood glucose and insulin indexes of type 2 diabetic rats.

\section{Chronic stress could improve blood lipid level in diabetic rat}

The levels of blood lipids, including FFA, TG, HDLC

Table 3 The blood lipid profile of each group

\begin{tabular}{lcccc}
\hline Group & FFA & TG & LDLC & HDLC \\
\hline Control group & $848.08 \pm 142.69$ & $95.29 \pm 15.58$ & $46.36 \pm 12.54$ & $17.67 \pm 12.54$ \\
Model group & $1,720.80 \pm 170.22^{\star \star}$ & $324.81 \pm 30.97^{\star *}$ & $71.47 \pm 12.73^{\star \star}$ & $9.98 \pm 8.36^{\star}$ \\
Model stress group & $1,295.20 \pm 201.59^{\#}$ & $232.85 \pm 26.03^{\# \#}$ & $63.39 \pm 12.55^{\#}$ & $14.92 \pm 6.94^{\#}$ \\
\hline
\end{tabular}

*, $\mathrm{P}<0.05 ;{ }^{* *}, \mathrm{P}<0.01$ compare to the control group;, $\mathrm{P}<0.05 ;{ }^{\# \#}, \mathrm{P}<0.01$ compared to the model group. FFA, free fatty acid; TG, triglyceride; HDLC, high-density lipoprotein cholesterol; LDLC, low-density lipoprotein cholesterol. 
A

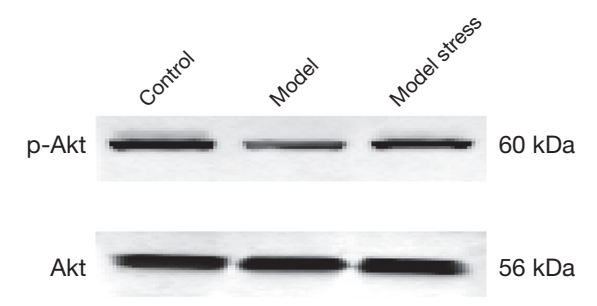

B

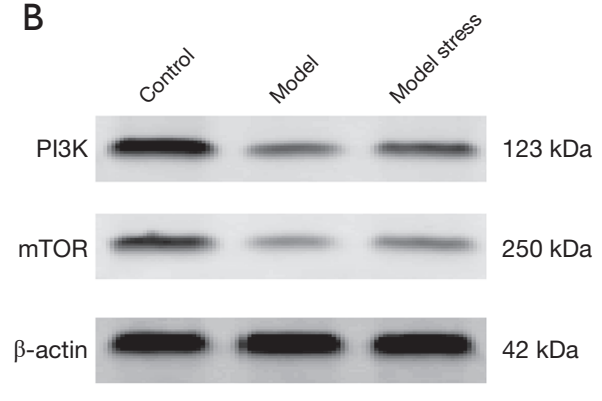

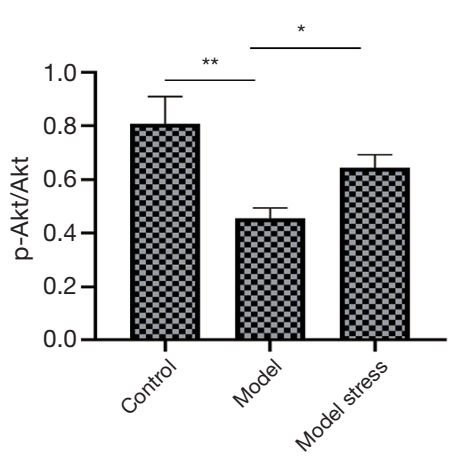

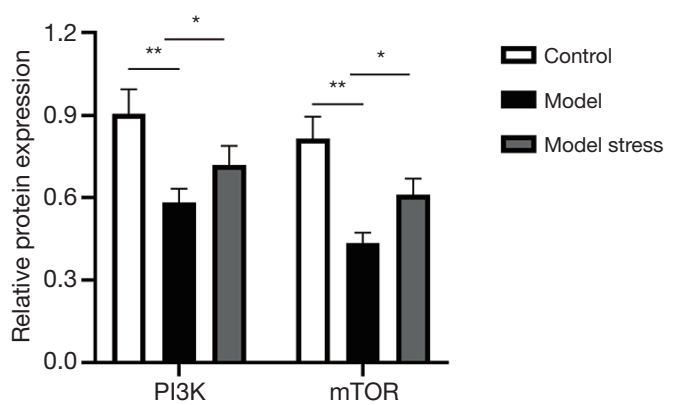

Figure 2 The protein levels of the pancreas in each group. (A) Expression of phosphorylated Akt in pancreas. (B) Expression of PI3K and mTOR in pancreas. *, $\mathrm{P}<0.05 ;{ }^{* *}, \mathrm{P}<0.01$.

and LDLC in the blood of SD rats in each group were detected. Our results showed that the content of FFA, TG, and LDLC in the model group was significantly higher than those in the control group $(\mathrm{P}<0.01)$, and the HDLC level of the model group was significantly lower than the control group $(\mathrm{P}<0.05)$. After seven-day forced swimming stimulation, it could be observed the levels of FFA, TG and LDLC were significantly decreased, and the HDLC level was increased in the blood of diabetic rats $(\mathrm{P}<0.05)$ (Table 3). In conclusion, 7-day forced swimming stimulation can improve the blood lipid levels of type 2 diabetes model rats to a certain extent.

\section{The effect of forced swimming stress on a pancreatic PI3K/ Akt signal pathway in diabetic rats}

To further explore the molecular mechanism of forced swimming stress in improving the physiological indexes of diabetic rats, we used the western blot to detect the protein levels of PI3K, Akt, and mTOR in the PI3K/ Akt signal pathway and the phosphorylation level of Akt (p-Akt) in each group of rats. The results showed that the protein levels of pancreatic PI3K, p-Akt, and mTOR in the diabetes model group were significantly lower than those protein levels in the control group $(\mathrm{P}<0.05)$, and there was no significant difference in the protein level of Akt. The protein levels of PI3K and mTOR and the phosphorylation level of Akt in the model stress group were significantly higher than those in the model group (Figure 2). The results indicated that one-week forced swimming stress could increase the protein levels of PI3K, p-Akt, and mTOR in the pancreas of diabetic rats, and play a specific role in improving the physiological indexes of diabetic rats.

\section{Discussion}

In this study, a type 2 diabetes model was established, and chronic forced swimming stimulation was given for one week to measure the effect of stress on the levels of blood glucose, blood lipid, and insulin, and the pancreatic PI3K/ Akt signal pathway was detected to explore its molecular mechanism further. These results suggest that chronic forced swimming stimulation could significantly improve the levels of blood glucose, blood lipid and serum insulin in diabetic model rats, and increase the protein level of PI3K, p-Akt, and mTOR in the pancreas of diabetic rats.

Studies have shown that stress can rapidly activate the hypothalamic-pituitary-suprarenal (HPA) axis and increase 
the release of glucocorticoids. Glucocorticoids have critical physiological functions related to diabetes, which can promote the mobilization of energy storage and thus induce the release of glucose and lipids into circulation. The release of glucocorticoids could suppress inflammation and increase blood pressure by stimulating the cardiovascular system and the sympathetic nervous system $(11,12)$. Stress includes exposure to stressful environments (including work stress and psychological stress) and emotional disorders (including depression and anxiety). In the case of type 2 diabetes (T2DM), the content of glucose or lipids excess the cellular energy requirements and constitute a metabolic pressure, which promotes insulin resistance over time (13). The biological response to stress may lead to type 2 diabetes, including cardiovascular disorders, functional neuroendocrine disorders including abnormal cortisol secretion, and increased inflammation. Currently, the effects of stress on patients with diabetes are not noticeably clear. Therefore, in this study, the diabetic model rats were given chronic forced swimming stress to discover the effect of chronic stress on diabetic model rats. The results of this study showed that one week of forced swimming stress could significantly reduce blood glucose and blood lipid levels and improve insulin indexes in diabetic model rats. These results may be caused by the rats being forced to do exercise for a long time, and exercise can promote the utilization of glucose and regulate glucose metabolism in type 2 diabetes (14). The study of Tang et al. also confirmed this point. Their results showed that exercise could improve glucose metabolism, and the mechanism may be related to the improvement of islet function by enhancing FKN expression through PI3K/Akt pathway (15). On the basis of these studies, our experiment added the detection of mTOR, FFA,TG, LDLC, HDLC, ISI and other indicators, which further proved that forced swimming stress improved the physiological indexes of diabetic model rats.As a component of the PI3K/Akt pathway and other cellular signals, mTOR is an important regulator of fuel metabolism and function in islet cells and immune cells. Thus, mTOR appears to have both antidiabetic and pro-diabetic effects. Our study shows that forced swimming can increase the expression of mTOR and improve the islet function in diabetic rats (16).

Studies by $\mathrm{Hu}$ et al. have found that sodium butyrate reduces type 2 diabetes by inhibiting the PERK-CHOP pathway in the endoplasmic reticulum (17). PPAR $\beta / \delta$ agonist GW501516 protects cells from lipid apoptosis and improves cell quality in type 2 diabetic rats by upregitating GPR40 and activating Akt/Bcl-2/Caspase-3 pathways (18). It has been found that swimming training can reduce insulin resistance in type 2 diabetic rats through $W n t 3 a / \beta$-catenin signaling pathway (19). Recent studies have shown that ten weeks of swimming training can significantly improve glucose tolerance and insulin resistance, reduce fasting blood glucose and decrease the insulin levels in diabetic model rats, suggesting that swimming training can improve insulin sensitivity and blood glucose regulation (20). In this study, we also showed that one-week forced swimming could improve the levels of blood glucose, blood lipids, and insulin in diabetic rats. The study on the diabetic rat model (21) shows that exercise can improve the PI3K activity of gastrocnemius muscle. The PI3K/Akt signal pathway has essential functions in the absorption and use of glucose and the regulation of blood glucose balance. Exercise can increase the sensitivity of skeletal muscle to insulin, promote the binding of insulin to the receptors of the muscle cell membrane, activate the phosphorylation and expression of PI3K/Akt signaling, cascade protein kinase, and regulate glucose metabolism (22-24). Also, the results of this study suggest that chronic forced swimming can activate the PI3K/Akt signal pathway and increase the protein levels of PI3K and mTOR and the phosphorylation level of Akt in the pancreas of diabetic rats, which may have a specific protective effect on type 2 diabetic rats.

\section{Acknowledgments}

Thank you very much for the technical support of Guangzhou Yujia Biological Technology Co., Ltd.

Funding: Shenzhen Key Medical Discipline Construction Fund.

\section{Footnote}

Reporting Checklist: The authors have completed the ARRIVE reporting checklist. Available at http://dx.doi. org/10.21037/atm-20-5304

Data Sharing Statement: Available at http://dx.doi. org/10.21037/atm-20-5304

Conflicts of Interest: All authors have completed the ICMJE uniform disclosure form (available at http://dx.doi. org/10.21037/atm-20-5304). The authors have no conflicts of interest to declare. 
Ethical Statement: The authors are accountable for all aspects of the work in ensuring that questions related to the accuracy or integrity of any part of the work are appropriately investigated and resolved. All animal experiments were performed in accordance with the guidelines for animal care and was approved by the Medical Ethics Committee of Shenzhen People's Hospital (No. LLKY-2020293).

Open Access Statement: This is an Open Access article distributed in accordance with the Creative Commons Attribution-NonCommercial-NoDerivs 4.0 International License (CC BY-NC-ND 4.0), which permits the noncommercial replication and distribution of the article with the strict proviso that no changes or edits are made and the original work is properly cited (including links to both the formal publication through the relevant DOI and the license). See: https://creativecommons.org/licenses/by-nc-nd/4.0/.

\section{References}

1. Cho NH, Shaw JE, Karuranga S, et al. IDF Diabetes Atlas: Global estimates of diabetes prevalence for 2017 and projections for 2045. Diabetes Res Clin Pract 2018;138:271-81.

2. Roden M, Shulman GI. The integrative biology of type 2 diabetes. Nature 2019;576:51-60.

3. Petersen KF, Dufour S, Befroy D, et al. Reversal of nonalcoholic hepatic steatosis, hepatic insulin resistance, and hyperglycemia by moderate weight reduction in patients with type 2 diabetes. Diabetes 2005;54:603-8.

4. Leung PS. Does vitamin D supplementation reduce type 2 diabetes risk? Ann Transl Med 2019;7:614.

5. Bellou V, Belbasis L, Tzoulaki I, et al. Risk factors for type 2 diabetes mellitus: An exposure-wide umbrella review of meta-analyses. PLoS One 2018;13:e194127.

6. Hackett RA, Steptoe A. Type 2 diabetes mellitus and psychological stress - a modifiable risk factor. Nat Rev Endocrinol 2017;13:547-60.

7. Shi L, Zhong LY, Shu Y. Effects of stress on related hormones of neuroendocrine in type 2 diabetic patients. Modern Medical Journal 2006.

8. Detke MJ, Rickels M, Lucki I. Active behaviors in the rat forced swimming test differentially produced by serotonergic and noradrenergic antidepressants. Psychopharmacology (Berl) 1995;121:66-72.

9. Islam MS, Loots du T. Experimental rodent models of type
2 diabetes: a review. Methods Find Exp Clin Pharmacol 2009;31:249-61.

10. Hongbing R. Research on animal model of type 2 diabetes mellitus. Journal of Hubei Institute For Nationalities (Medical Edition) 2013;30:78-81.

11. Brotman DJ, Golden SH, Wittstein IS. The cardiovascular toll of stress. Lancet 2007;370:1089-100.

12. Ulrich-Lai YM, Herman JP. Neural regulation of endocrine and autonomic stress responses. Nat Rev Neurosci 2009;10:397-409.

13. McEwen BS, Wingfield JC. The concept of allostasis in biology and biomedicine. Horm Behav 2003;43:2-15.

14. Frosig C, Rose AJ, Treebak JT, et al. Effects of endurance exercise training on insulin signaling in human skeletal muscle: interactions at the level of phosphatidylinositol 3-kinase, Akt, and AS160. Diabetes 2007;56:2093-102.

15. Tang $Y$, Tan Z, Qin X. Effects of Swimming Training on Factalkine Expression and P13K/Akt Signal Pathway in Pancreas of Type 2 Diabetic Rats. Wuhan Tiyu Xueyuan Xuebao 2014;48:97-100.

16. Tuo Y, Xiang M. mTOR: A double-edged sword for diabetes. J Leukoc Biol 2019;106:385-95.

17. Hu Y, Liu J, Yuan Y, et al. Sodium butyrate mitigates type 2 diabetes by inhibiting PERK-CHOP pathway of endoplasmic reticulum stress. Environ Toxicol Pharmacol 2018;64:112-21.

18. Li J, Xu S, Liu Y, et al. Activated PPARbeta/delta Protects Pancreatic beta Cells in Type 2 Diabetic GotoKakizaki Rats from Lipoapoptosis via GPR40. Lipids 2019;54:603-16.

19. Yang $Q$, Wang WW, Ma $P$, et al. Swimming training alleviated insulin resistance through $\mathrm{Wnt} 3 \mathrm{a} /$ beta-catenin signaling in type 2 diabetic rats. Iran J Basic Med Sci. 2017;20:1220-26.

20. Ghiasi R, Ghadiri Soufi F, Somi MH, et al. Swim Training Improves HOMA-IR in Type 2 Diabetes Induced by High Fat Diet and Low Dose of Streptozotocin in Male Rats. Adv Pharm Bull 2015;5:379-84.

21. Krisan AD, Collins DE, Crain AM, et al. Resistance training enhances components of the insulin signaling cascade in normal and high-fat-fed rodent skeletal muscle. J Appl Physiol (1985) 2004;96:1691-700.

22. Luciano E, Carneiro EM, Carvalho CR, et al. Endurance training improves responsiveness to insulin and modulates insulin signal transduction through the phosphatidylinositol 3-kinase/Akt-1 pathway. Eur J Endocrinol 2002;147:149-57. 


\section{Page 8 of 8}

23. Langfort J, Viese M, Ploug T, et al. Time course of GLUT4 and AMPK protein expression in human skeletal muscle during one month of physical training. Scand J Med Sci Sports 2003;13:169-74.

24. Thurmond DC, Pessin JE. Molecular machinery involved

Cite this article as: Li L, He D, Jiang K, Zhao Y. Effects of forced swimming stress on expression and phosphorylation of PI3K/Akt signal pathway in pancreas of type 2 diabetic rats. Ann Transl Med 2020;8(16):1006. doi: 10.21037/atm-20-5304
Li et al. Forced swimming stress and type 2 diabetic rats

in the insulin-regulated fusion of GLUT4-containing vesicles with the plasma membrane (review). Mol Membr Biol 2001;18:237-45.

(English Language Editor: J. Chapnick) 